\title{
Strength Training in Older Adults: Changes in Mechanical Muscle Function And Functional Performance
}

\author{
Paolo Caserotti ${ }^{1,2, *}$ \\ ${ }^{1}$ Laboratory of Epidemiology, Demography, and Biometry, National Institute on Aging, Bethesda, USA \\ ${ }^{2}$ Institute of Sports Science and Clinical Biomechanics, University of Southern Denmark, Denmark
}

\begin{abstract}
Aging is associated with a loss of skeletal muscle mass, increase in intramuscular fat and neural changes which result in reduced mechanical muscle function and impaired functional performance. Rapid mechanical muscle function (i.e. muscle power, rate of force development) and power-based time-constrained functional motor tasks such as reversing an impending fall are more affected by aging than muscle strength and strength-based functional motor tasks. Strength training has been consistently effective to reduce neuromuscular deterioration even at very old age by evoking muscle hypertrophy, muscle strength and neural improvements, although changes in muscle power have not always been observed. Power training, a novel type of strength training has recently been shown to be highly effective and potentially superior to traditional strength training for muscle power and functional performance improvements.
\end{abstract}

This brief article will review key literature and provide a detailed example of a specific type of power training in old and very old adults.

Keywords: Power and strength training, aging, functional performance.

\section{INTRODUCTION}

It is well known that the aging process is associated with loss of skeletal muscle mass and increase in intramuscular fat, the latter also defined as muscle attenuation $[1,2]$. Decline in muscle mass is caused by a general loss of muscle fibers and reduction in cross-sectional area of the surviving fibers, with the fast-twitch fibers showing accelerated muscle atrophy, especially at advanced age [3]. Additionally, qualitative changes occur in the old muscle: specific tension (force normalized to cross-sectional area) of whole muscle and of both type I and type II muscle fibers is lower compared to a younger muscle and maximal shortening velocity is reduced [4].

Taken together, the combined results of quantitative and qualitative muscle changes determine a progressive decline in the overall mechanical muscle function, with muscle power and explosive muscle force declining at a steeper rate than muscle strength $[5,6]$. From a functional standpoint, these changes make it progressively harder to carry out daily motor activities (e.g. level walking, chair rising, carrying shopping bags) due to reduced functional capacity and higher relative effort for each motor task [7, 8]. Importantly, although motor performance in general becomes impaired with increasing age, time-constrained and rapid motor tasks (i.e. 200-300 ms duration) such as balance recovery after tripping may become even more challenged for older adults [9].

\footnotetext{
*Address to this corresspondence at the Suite 3C309 Gateway Building 7201 Wisconsin Avenue Bethesda, USA; 20892-9205; Tel: 301-496-1178, 301-496-6524; Fax: 301-496-4006;

E-mail caserottip@mail.nih.gov
}

\section{MECHANICAL MUSCLE FUNCTION WITH AGING: MUSCLE STRENGTH, MUSCLE POWER AND EX- PLOSIVE FORCE}

The relationship between muscle strength and muscle mass is relatively well known and it is primarily related to the number of active sarcomeres in parallel [10-13]. Although a close association between decline in muscle mass with reduced muscle strength with aging has been identified [14], a disproportionate greater loss of muscle strength compared to muscle mass was recently reported in a longitudinal study ( $\sim 3 \%$ versus $\sim 1 \%$ per year, respectively) [2]. Importantly, low muscle mass, muscle attenuation and muscle strength have been independently associated with increased risk of mobility limitation, but the association of muscle mass with mobility limitations seems dependent of low muscle strength [15].

Although muscle strength has been recognized as an important predictor for reduced functional performance [15], emerging evidence suggests that muscle power (the product of force time velocity or the rate of performing work) may play a more important role for loss of functional independence, incidence of falling and functional motor performance [6, 16-18].

Muscle power requires the integration of muscle force with the ability to develop contraction velocity, the latter related to the number of active sarcomeres in series $[12,13]$. Thus, although muscle power shares several muscle properties with muscle strength (e.g. physiological muscle crosssectional area), it is influenced by numerous additional neuromuscular properties (e.g. fiber type composition, muscle fiber length, muscle fiber pennation angle, motor unit firing 
frequency, discharge doublets) [13]. Additionally, muscle power seems to be maximized at a specific external resistance [19]; that is, maximum muscle power is obtained when muscles contract against a load intensity ranging between $\sim 40$ and $\sim 70 \%$ of 1Repetition Maximum.

Explosive force (rate of force development, RFD) is intimately linked to muscle power as it determines the magnitude of acceleration in the initial phase of a movement and thereby influence movement velocity [20, 21]. RFD is the most time-dependent mechanical muscle variable, essential for short-lasting movement (e.g. preventing an impending fall) or with movements with limited range of motion (e.g. ankle extension). In older subjects, RFD has been reported to correlate with postural control ability [22] and with poor balance recovery after tripping [23].

Importantly, the association of RFD and muscle power may vary according to the bio-physical determinants of the motor task evaluated (e.g. range of motion, time available for power generation, power-load relationship) [21].

\section{STRENGTH/POWER TRAINING FOR OLDER ADULTS}

Most strength training interventions in older adults have implemented strength training regimes with relatively slow movement velocity and high training intensity [24]. Heavyresistance strength training protocols have been effective in eliciting muscle hypertrophy according to the dose-response relationship that dictates greater hypertrophy at heavy loadings (>75\% 1RM) [24]. Older adults also maintain the ability to develop muscle hypertrophy in response to heavyresistance strength training $(80 \% 1 \mathrm{RM})$, which is generally accompanied by muscle strength improvements [25, 26]. Similarly, heavy-resistance training also leads to enhanced neural function in old [25, 27] and very old individuals [28]. This is reflected by elevated agonist EMG activity [27], decreased antagonist muscle activation [27] and diminished muscle activation deficit $[29,30]$, albeit the latter not consistently [31]. Improvements in muscle strength following strength training protocols are in general consistent, but the effect on muscle power seems more blurred $[17,18]$.

The acknowledgement that muscle power is a key component of time-constrained and power-based functional performances and essential in critical situations such as impending falls and postural adjustments has recently resulted in recommendation suggesting that strength training programs for older adults should be designed to maximize muscle power [32].

Power training is a specific type of strength training, in general designed by manipulating traditional strength training variables and primarily movement velocity and training intensity. The rationale for this differentiation from a traditional strength training protocol is that movement velocity or/and the intention to maximally accelerate the training load may be particularly relevant for muscle power and powerbased functional performance improvements for older adults [12, 33-35]. Although, the key component of power training protocols is "maximal intentional velocity", large variation in training intensity (from $40 \%$ to $80 \%$ 1RM) has been experimented in older adults.
The effect of power versus strength training has been investigated in few recent studies [36-41].

Power training was reported to be superior in increasing muscle power compared with heavy-resistance training in elderly women and men with self-reported disability performing similar training at 70\% 1RM $[36,39]$ and in healthy older men training at lower load intensity (60\% 1RM) [40]. Importantly, in the latter study, power training improved functional performance (e.g. repeated chair rise) while no changes occurred with traditional strength training, along with similar muscle strength increase in both groups [40]. Additionally, power was increased to a larger extent in healthy older women when using isokinetic training at high versus low velocity [42]. Amplified training-related responses in maximal muscle strength and similar increase in muscle power were recently demonstrated with power training protocols designed with heavy $(80 \% 1 \mathrm{RM})$ compared to moderate $(50 \% 1 \mathrm{RM})$ intensity using pneumatic training equipment in healthy 69-year old adults. This may indicate the need of high loading intensity to improve concomitantly strength and power [37]. Contrarily to the above findings, a recent study reported similar increase in muscle power and strength using either traditional slow strength training $(75 \%$ $1 \mathrm{RM}$ ) or fast varied strength training (3 sets, $45 \%, 60 \%$ and $75 \%$ 1RM) [41].

The reason for the discrepancy between studies may depend on several factors, including different training protocols used (e.g. \% 1RM for training intensity, isoinertial versus mechanically braked training equipment), age-groups, and tests used to assess muscle power/strength and functional performance. Thus, despite growing evidence suggesting superior effect of power versus strength training protocols, full consensus has not been reached yet. Importantly, the superior effect on functional performance of one versus the other training regime likely depends on specific performance biophysical characteristics, and for example whether this has time-constraints, limited range of motion and whether it requires maximal effort (primarily strength-based) or maximal velocity (primarily power-based).

\section{EXPLOSIVE-TYPE HEAVY-RESISTANCE STRENGTH TRAINING}

To address some of the above questions we conducted a series of experiments by designing a training protocol defined as explosive-type heavy-resistance strength training which combined some of the traditional strength and power training components. The aim was to investigate the effect of this training protocol on mechanical muscle function and functional performance in old and very old elderly women. Our primary outcomes were explosive muscle force and muscle power.

Details on selection of subjects has been published previously [21]. In short, two age-groups of elderly women, group 60 (mean age 62.8 SD 2.2, $n=34$ ) and group 80 (mean age 81.8 SD 2.2, $\mathrm{n}=24$ ) without any previous experience on strength training were selected. The experiments included a cross-sectional study aimed at identifying the age-associated differences in selected mechanical muscle function including explosive muscle force, maximal strength and muscle power 
and selected functional performances. Additionally, we performed an intervention study where the two age-groups were divided respectively into training (TG80 and TG60) and control (CG80 and CG60) groups. TG80 and TG60 underwent a 12-week explosive-type heavy resistance strength training program.

\section{Measurements}

\section{Explosive Muscle Force, Maximal Muscle Strength and EMG Measurements}

Explosive muscle force (rate of force development, RFD) and maximal muscle strength (MVC) were assessed during a maximal isometric unilateral leg press in a custom-built device [21]. Subjects pushed as hard and fast as possible with their dominant leg against a fixed instrumented footplate (Kistler 9367/8 B). Force signal was synchronized with EMG signals and sampled at $1 \mathrm{KHz}$. Pairs of surface electrodes (Blue Sensor, Ambu, Denmark) were positioned at the quadriceps [Vastus Lateralis (VL), Vastus Medialis (VM), Rectus Femoris (RF)] and hamstring muscles [Biceps Femoris (BF), Semitendinosus (ST)]. Contractile RFD was determined in the trial with highest peak force. RFD was calculated as the mean tangential slope of the force-time curve in the initial 100, 200, 300 and $400 \mathrm{~ms}$ after onset of contraction (RFD $=\Delta$ force $/ \Delta$ time $)[43]$.

\section{Muscle Power}

Muscle power was assessed by i) single leg press (Nottingham power rig) (NPR); ii) weight-bearing multijoint mo- tor task (Countermovement jump, CMJPpeak) on a force plate (Kistler 9281B) [30]. Additionally, CMJ jump height (CMJ JH) was assessed.

\section{Functional Performance}

Functional performance included five timed repeated chair-rise, physical performance test battery (PPT), maximal and self-selected $10-\mathrm{m}$ walking speed.

\section{Training Protocol}

The training program was aimed at maximizing explosive muscle force and muscle power and was designed combining the principles of heavy-resistance strength training with power training. The training consisted of a 12 -week progressive explosive-type heavy-resistance strength training program, twice a week with at least two days between training sessions. Training was performed exclusively for the lower limbs (bilateral knee extension, horizontal leg press, hamstring curls, calf rise, and inclined leg press) using isoinertial resistance training equipment (cybex) [21]. This equipment allows for unrestricted acceleration of the training load and thereby enables to achieve variable velocity throughout the entire range of motion. Four sets were performed for each exercise with training loads of $75-80 \%$ 1RM (8-10 repetitions per set) [44]. Explosive movement pattern (i.e. maximal intentional load acceleration) was employed during the concentric contraction while the eccentric phase of each exercise was performed using and slow-to-moderate speed. Training loads were adjusted every 2 weeks using the 4-8 repetition method [44].

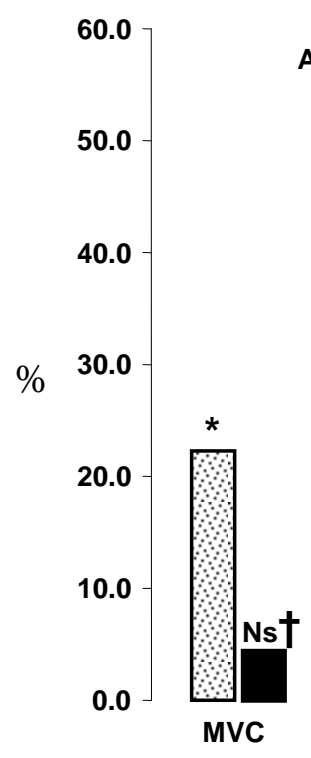

A

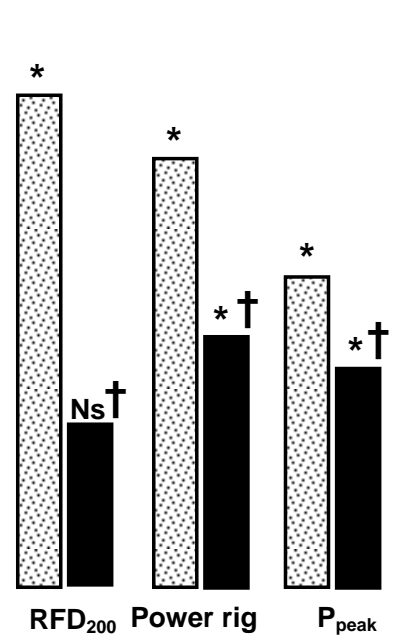

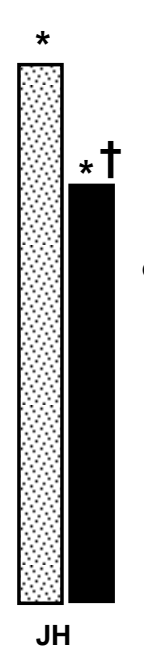

Diff. between TG80 and CG60 at baseline.

Diff. betweenTG80 post power training and CG60 post no activity.

20.0

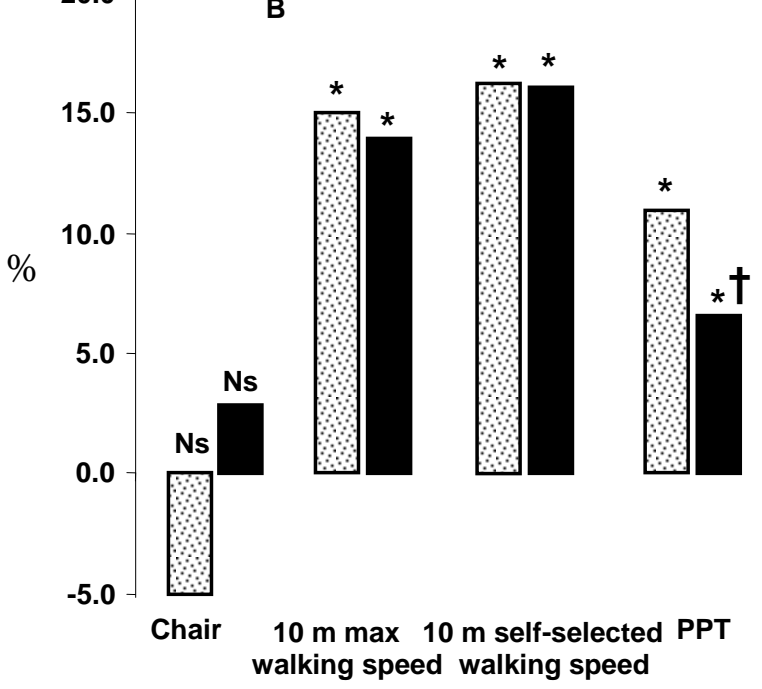

Fig. (1). A and B. Comparison between the training group 80 (TG80) and the control group 60 (CG60). The Fig. (1A) illustrates the age-related deficit in maximal voluntary contraction (MVC), rate of force development at $200 \mathrm{~ms}$ (RFD200), mean power during the power rig, concentric peak power (Ppeak), maximal jump height (JH). Fig. (1B) illustrates the age-related deficit in chair-rise, maximal walking speed, self-seceted walking speed and Physical Performance Battery test. The Dotted bars represent the difference in percent (deficit) between the training group 80 (TG80) and the control group 60 (CG60) at baseline. Filled bars represent the deficit in percent between these groups after 12 weeks of explosive-type heavy-resistance strength training for TG80 and 12 weeks of unchanged life style for CG60.

* Represents significant difference in percent (deficit) in TG80 relative to CG60 $(\mathrm{P}<0.05)$; $\dagger$ Represents significant reduction in this deficit with training $(\mathrm{P}<0.05)$; Ns indicates no significant difference between the two groups, i.e. full removal of the age-related deficit $(\mathrm{P}<0.05)$. Adapted from Caserotti et al., 2008 [21]. 


\section{Results}

\section{Rate of Force Development, Muscle Strength and Muscle Activation}

The increase in RFD following training ranged, according to different time intervals, between $41.7 \%$ to $58.5 \%$ $(\mathrm{P}<0.05)$ for $\mathrm{TG} 80$ and $11.8 \%$ and $18.2 \% \quad(\mathrm{P}<0.05)$ for TG60. Specific RFD (RFD divided by MVC) increased significantly only in TG80 (between $31.0 \%$ and $43.3 \%$ ), indicating a qualitative change in rapid contractile force production (RFD). MVC increased by $28.1 \%$ (TG80) and by $21.5 \%$ (TG60) $(\mathrm{P}<0.05)$. Changes in RFD and MVC were accompanied by significant increased EMG values for the Vastus medialis (all time windows) only for the oldest group (TG80).

\section{Muscle Power}

Muscle power as measured by NPR increased by $28 \%$ (TG80) and 14\% (TG60) $(\mathrm{P}<0.05)$. CMJ JH increased in both groups $(18.1 \%$ and $9.7 \%$ for TG80 and TG60, respectively) $(\mathrm{P}<0.05)$ while CMJPpeak increased significantly only for TG60 and had a trend for TG80.

\section{Functional Performance}

TG80 improved repeated chair rise by $12 \%$ and PPT by $6.5 \%(\mathrm{P}<0.05)$, while no changes occurred for the $10-\mathrm{m}$ maximal and self-selected walking speed. TG60 improved only for the repeated chair-rise by $11.5 \%(\mathrm{P}<0.05)$.

\section{CONCLUSION}

Explosive-type heavy-resistance strength training, a novel type of strength training for very old elderly people, was highly effective to elicit substantial improvements in maximal mechanical muscle function (rapid force generation, muscle power and muscle strength) and in functional performance in old and very old women. The improvements in the in MVC and rapid force for the oldest group (TG80) were of such a magnitude that the deficit reported at baseline of TG80 relative to the younger control group (CG60) disappeared (i.e. statistically non-significant) after training (Fig. 1). These improvements were accompanied by morphological and neural adaptations with a significant muscle hypertrophy especially for the quadriceps in both age-groups (data not shown), and increased muscle activation (EMG) in the oldest group (TG80). This training type seems to be safe and promising for reducing the accelerated age-related decline in rapid muscle force and muscle power which is one of the most threatening aspects of the neuromuscular aging process for its implication with fall prevention and risk of functional dependency. Although dose-response was not investigated, being a relatively low training frequency (twice a week) and short intervention period (12 weeks), this training was able to induce changes in the oldest group of a significant magnitude able to represent a potential rejuvenation of the oldest old.

Finally, it is important to consider that this training was investigated in relatively healthy elderly subjects and results may not be generalized to older adults with different health and functional conditions.

\section{FINAL REMARKS}

Since the classical study of Fiatarone (1990) [45], which opened a new window into resistance training methodology for older adults by demonstrating the trainability of frail older adults with high intensity loading, a vast number of studies have successfully incorporated this methodological aspect into their training regimes. Indeed, it is becoming progressively clearer that older adults can well tolerate "heavy training regimes" (e.g. power training, speed training) that were once exclusively designated to athletic and younger populations and which are able to elicit substantial improvements in mechanical muscle function. In 2008, the US government for the first time included as a part of their recommendation for physical activity in the general population and especially in the older adults power training as a separate regimes from strength training [46]. While there seems to be no doubt that strength training is in general highly effective in inducing muscle strength adaptation, improvements in muscle power following strength training protocols are being questioned. Power training, seems to be indeed a novel and promising training regime for older adults and potentially superior to traditional strength training.

Nonetheless, further research is needed to better address the effect of these novel training regimes on functional outcomes (e.g. walking speed, chair-rise) and especially on time-constrained functional outcomes highly dependent on rapid muscle contraction such as fall prevention and postural control. Finally, issues such as dose-response, optimal training loading, combined effect of strength/ power training with other training regimes (e.g. aerobic, balance), still need to be further addressed for older adults in general and for specific subgroups such as frail older adults.

\section{ACKNOWLEDGEMENT}

This research was supported in part by the Intramural Research Program of the NIH, National Institute on Aging.

\section{REFERENCE}

[1] Goodpaster BH, Carlson CL, Visser M, et al. Attenuation of skeletal muscle and strength in the elderly: the Health ABC Study. J Appl Physiol 2001; 90(6): 2157-65.

[2] Goodpaster BH, Park SW, Harris TB, et al. The loss of skeletal muscle strength, mass, and quality in older adults: the health, aging and body composition study. J Gerontol A Biol Sci Med Sci 2006; 61(10): 1059-64.

[3] Andersen JL. Muscle fibre type adaptation in the elderly human muscle. Scand J Med Sci Sports 2003; 13(1): 40-7.

[4] Yu F, Hedstrom M, Cristea A, Dalen N, Larsson L. Effects of ageing and gender on contractile properties in human skeletal muscle and single fibres. Acta Physiol (Oxf) 2007; 190(3): 229-41.

[5] Skelton DA, Greig CA, Davies JM, Young A. Strength, power and related functional ability of healthy people aged 65-89 years. Age Ageing 1994; 23(5): 371-7.

[6] Skelton DA, Kennedy J, Rutherford OM. Explosive power and asymmetry in leg muscle function in frequent fallers and nonfallers aged over 65. Age Ageing 2002; 31(2): 119-25.

[7] Hortobagyi T, Mizelle C, Beam S, DeVita P. Old adults perform activities of daily living near their maximal capabilities. J Gerontol A Biol Sci Med Sci 2003; 58(5): M453-M460.

[8] Ploutz-Snyder LL, Manini T, Ploutz-Snyder RJ, Wolf DA. Functionally relevant thresholds of quadriceps femoris strength. J Gerontol A Biol Sci Med Sci 2002; 57(4): B144-B152.

[9] Pijnappels M, Bobbert MF, van Dieen JH. Control of support limb muscles in recovery after tripping in young and older subjects. Exp Brain Res 2005; 160(3): 326-33.

[10] Reed RL, Pearlmutter L, Yochum K, Meredith KE, Mooradian AD. The relationship between muscle mass and muscle strength in the elderly. J Am Geriatr Soc 1991; 39(6): 555-61.

[11] Metter EJ, Lynch N, Conwit R, et al. Muscle quality and age: cross-sectional and longitudinal comparisons. J Gerontol A Biol Sci Med Sci 1999; 54(5): B207-B218. 
[12] Kraemer WJ, Newton RU. Training for muscular power. Phys Med Rehabil Clin N Am 2000; 11(2): 341-68, vii.

[13] Edgerton VR, Roy RR, Gregor RJ, Rugg S. In: Jones NL, McCartney N, McComas AJ, Eds. Human Kinehics, Champaign III, Human muscle power. 1986; pp. 43-64.

[14] Frontera WR, Hughes VA, Fielding RA, et al. Aging of skeletal muscle: a 12-yr longitudinal study. J Appl Physiol 2000; 88(4): 1321-6.

[15] Visser M, Goodpaster BH, Kritchevsky SB, et al. Muscle mass, muscle strength, and muscle fat infiltration as predictors of incident mobility limitations in well-functioning older persons. J Gerontol A Biol Sci Med Sci 2005; 60(3): 324-33.

[16] Foldvari M, Clark M, Laviolette LC, et al. Association of muscle power with functional status in community-dwelling elderly women. J Gerontol A Biol Sci Med Sci 2000; 55(4): M192-M199.

[17] Bean JF, Leveille SG, Kiely DK, et al. A comparison of leg power and leg strength within the InCHIANTI study: which influences mobility more? J Gerontol A Biol Sci Med Sci 2003; 58(8): 72833.

[18] Suzuki T, Bean JF, Fielding RA. Muscle power of the ankle flexors predicts functional performance in community-dwelling older women. J Am Geriatr Soc 2001; 49(9): 1161-7.

[19] Macaluso A, De Vito G. Comparison between young and older women in explosive power output and its determinants during a single leg-press action after optimisation of load. Eur J Appl Physiol 2003; 90(5-6): 458-63.

[20] Aagaard P, Simonsen EB, Andersen JL, Magnusson P, DyhrePoulsen P. Increased rate of force development and neural drive of human skeletal muscle following resistance training. J Appl Physiol 2002; 93(4): 1318-26.

[21] Caserotti P, Aagaard P, Larsen JB, Puggaard L. Explosive heavyresistance training in old and very old adults: changes in rapid muscle force, strength and power. Scand J Med Sci Sports 2008; 18(6): 773-82.

[22] Izquierdo M, Aguado X, Gonzalez R, Lopez JL, Hakkinen K. Maximal and explosive force production capacity and balance performance in men of different ages. Eur J Appl Physiol Occup Physiol 1999; 79(3): 260-7.

[23] Pijnappels M, Bobbert MF, van Dieen JH. Push-off reactions in recovery after tripping discriminate young subjects, older nonfallers and older fallers. Gait Posture 2005; 21(4): 388-94.

[24] Fry AC. The role of resistance exercise intensity on muscle fibre adaptations. Sports Med 2004; 34(10): 663-79.

[25] Suetta C, Aagaard P, Rosted A, et al. Training-induced changes in muscle CSA, muscle strength, EMG, and rate of force development in elderly subjects after long-term unilateral disuse. J Appl Physiol 2004; 97(5): 1954-61.

[26] Hakkinen $\mathrm{K}$, Kallinen M, Izquierdo $\mathrm{M}$, et al. Changes in agonistantagonist EMG, muscle CSA, and force during strength training in middle-aged and older people. J Appl Physiol 1998; 84(4): 1341-9.

[27] Ferri A, Scaglioni G, Pousson M, et al. Strength and power changes of the human plantar flexors and knee extensors in response to resistance training in old age. Acta Physiol Scand 2003; 177(1): 69-78

[28] Hakkinen K, Newton RU, Gordon SE, et al. Changes in muscle morphology, electromyographic activity, and force production characteristics during progressive strength training in young and older men. J Gerontol A Biol Sci Med Sci 1998; 53(6): B415B423.

[29] Suetta C, Hvid LG, Justesen L, et al. Effects of aging on human skeletal muscle after immobilization and retraining. J Appl Physiol 2009; 107(4): 1172-80.

[30] Harridge SD, Kryger A, Stensgaard A. Knee extensor strength, activation, and size in very elderly people following strength training. Muscle Nerve 1999; 22(7): 831-9.

[31] Klass M, Baudry S, Duchateau J. Voluntary activation during maximal contraction with advancing age: a brief review. Eur J Appl Physiol 2007; 100(5): 543-51.

[32] Evans WJ. Exercise strategies should be designed to increase muscle power. J Gerontol A Biol Sci Med Sci 2000; 55(6): M309M310.

[33] Porter MM. Power training for older adults. Appl Physiol Nutr Metab 2006; 31(2): 87-94.

[34] Sayers SP. High-speed power training: a novel approach to resistance training in older men and women: a brief review and pilot study. J Strength Cond Res 2007; 21(2): 518-26.

[35] Hazell T, Kenno K, Jakobi J. Functional benefit of power training for older adults. J Aging Phys Act 2007; 15(3): 349-59.

[36] Fielding RA, LeBrasseur NK, Cuoco A, et al. High-velocity resistance training increases skeletal muscle peak power in older women. J Am Geriatr Soc 2002; 50(4): 655-62.

[37] de Vos NJ, Singh NA, Ross DA, et al. Optimal load for increasing muscle power during explosive resistance training in older adults. J Gerontol A Biol Sci Med Sci 2005; 60(5): 638-47.

[38] Signorile JF, Carmel MP, Czaja SJ, et al. Differential increases in average isokinetic power by specific muscle groups of older women due to variations in training and testing. J Gerontol A Biol Sci Med Sci 2002; 57(10): M683-M690.

[39] Marsh AP, Miller ME, Rejeski WJ, Hutton SL, Kritchevsky SB. Lower extremity muscle function after strength or power training in older adults. J Aging Phys Act 2009; 17(4): 416-43.

[40] Bottaro M, Machado SN, Nogueira W, Scales R, Veloso J. Effect of high versus low-velocity resistance training on muscular fitness and functional performance in older men. Eur J Appl Physiol 2007; 99(3): 257-64.

[41] Henwood TR, Riek S, Taaffe DR. Strength versus muscle powerspecific resistance training in community-dwelling older adults. J Gerontol A Biol Sci Med Sci 2008; 63(1): 83-91.

[42] Signorile JF, Carmel MP, Lai S, Roos BA. Early plateaus of power and torque gains during high- and low-speed resistance training of older women. J Appl Physiol 2005; 98(4): 1213-20.

[43] Aagaard P, Simonsen EB, Andersen JL, Magnusson P, DyhrePoulsen P. Neural adaptation to resistance training: changes in evoked V-wave and H-reflex responses. J Appl Physiol 2002 92(6): 2309-18.

[44] Baechle TR, Earle RW, Wathen D. In: Baechle TR, Earle RW, Eds. Essentials of strength training and conditioning. Human Kinetics Champaign, IL, 2000; pp. 395-426.

[45] Fiatarone MA, Marks EC, Ryan ND, et al. High-intensity strength training in nonagenarians. Effects on skeletal muscle. JAMA 1990; 263(22): 3029-34.

[46] Physical Activity Guidelines for Americans 2008. Available at: www. health govlpaguidelines

This is an open access article licensed under the terms of the Creative Commons Attribution Non-Commercial License (http://creativecommons.org/licenses/by-nc/3.0/) which permits unrestricted, non-commercial use, distribution and reproduction in any medium, provided the work is properly cited. 